MATEC Web of Conferences 11,02004 (2014)

DOI: $10.1051 /$ matecconf / 20141102004

(C) Owned by the authors, published by EDP Sciences, 2014

\title{
PERFORMANCE STUDY OF CONCRETE-FILLED STEEL TUBES
}

\author{
A. Chair ${ }^{1}$, A. Djebbar ${ }^{1}$, N. Djebbar ${ }^{1}$ \\ ${ }^{1}$ LMDC, Civil Engineering Département, Constantine 1 University, Algeria
}

\begin{abstract}
Abstract. Concrete-filled steel tubes circular section columns are increasingly used in the design of modern multi- stories structures. Investigations developed in recent decades confirm their beneficial use. They are used as a structural component system, given their excellent energy absorption and dissipation, or as a rehabilitation tool for recovery of damaged components after a seismic event. They contribute through their performance to confer high strength to elements, as high ductility and high rigidity, with a remarkable optimization of the used materials.

The main objective of this work is to review the principal models characterizing the behavior laws of concrete confined by steel jacket. An analysis based on Samantha model was conducted to highlight the parameters affecting the sectional behavior, and to evaluate the gains in strength and deformation. A section strength capacity investigation is presented. Results are compared with some codes recommendations.

This type of structure can be used in Algeria for rehabilitating old buildings, or strengthening reinforced concrete columns to improve their structural performance. However, new regulatory measures should be considered in order to introduce this compound element in the construction.
\end{abstract}

\section{Introduction}

Les poteaux circulaires composites en tubes d'acier remplis de béton sont de plus en plus utilisés dans la conception des structures modernes multi-étagées (tours). Les investigations développées ces dernières décennies confirment leur emploi bénéfique comme élément structurel qui présente de bonne performance du point de vue résistance, ductilité et rigidité, avec une optimisation remarquable des matériaux mis en œuvre. En plus de ces avantages, les tubes en acier servent aussi de coffrage, réduisant ainsi le coût et la durée de réalisation. Ils constituent les éléments principaux développant la capacité de résistance pour reprendre les charges verticales et servir de contreventement pour équilibrer les charges latérales.

Une série d'études est présentée dans ce contexte [Tomi 1991; Liang et Uy 2000; Susantha et al. 2001; Bradford, Loh, et Uy 2002, Huang et al. 2002, Morino et Tsuda 2002; $\mathrm{Hu}$ et al. 2003; Han 2004; Ehab et Young 2006]. Les résultats d'essais expérimentaux existants ont étés passés en revue afin de mieux cerner les principaux paramètres influant sur les lois de comportement du matériau: diamètre du poteau, épaisseur du tube d'acier, résistance du béton et limite élastique de l'acier.

L'objectif principal de ce travail est de présenter une synthèse des différents modèles caractérisant les lois de comportement du béton confiné par chemise en acier. Une analyse articulée sur le modèle de Susantha ${ }^{2}$, a été menée pour faire ressortir les facteurs prédominants sur le comportement sectionnel, et pouvoir évaluer les gains en résistance et en déformation. Une investigation relative à la capacité de résistance de la section est développée, et les résultats obtenus ont été comparés avec certains codes en l'occurrence Euro $4^{9}$ et $\mathrm{ACI}^{10}$.

\section{Observations expérimentales}

Les figures 1, 2 présentent les relations $\sigma-\varepsilon$ découlant des résultats expérimentaux de Giakoumelis ${ }^{10}$ et Tomii ${ }^{11}$, sur des spécimens en tubes d'acier circulaires remplis de béton sous chargement monotone axial, où les principaux paramètres sont mis en évidence.

Essai sur machine universelle : le comportement charge déformation de la région test du poteau est enregistré à travers deux capteurs potentiométriques de déplacement (LVDT) placés sur deux faces opposées du poteau. La charge étant appliquée selon une incrémentation qui lui correspond à chaque fois une série de lectures (déformation longitudinale et transversale mesurées à partir des jauges électriques). L'incrémentation de la charge dans la région inélastique est tributaire des déformations.

Use A4 paper size $(210 \times 297 \mathrm{~mm})$ and adjust the margins to those shown in Table 1 . The final printed area will be $172 \times 252 \mathrm{~mm}$.

\subsection{Effet de $f_{\text {co }}$}

La figure 1 montre l'influence de la résistance du béton sur le comportement $\sigma-\varepsilon$ du spécimen. On constate que la rigidité initiale (pente des diagrammes) est non affectée par $f_{\text {co }}$, alors que la capacité de déformation s'en trouve réduite. 


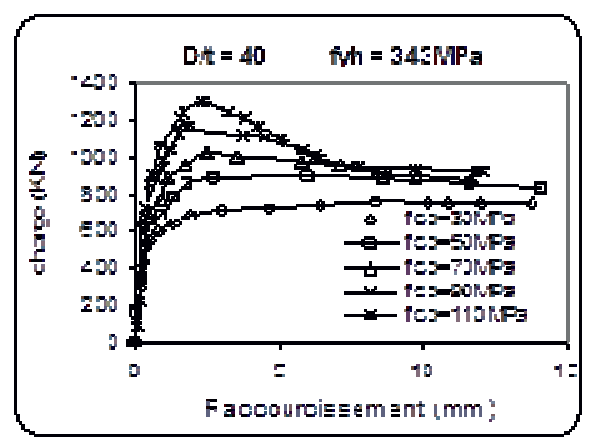

Fig. 1. Effet de la résistance du béton $\mathrm{f}_{\text {co }}$

\subsection{Effet du rapport $D / t$}

La figure 2 montre l'influence $\mathrm{du}$ rapport $\mathrm{D} / \mathrm{t}$ (diamètre sur l'épaisseur du tube d'acier) selon la courbe charge-raccourcissement longitudinal de l'élément. On constate que la rigidité initiale est toujours non affectée, car l'intervention du confinement à ce stade reste négligeable. La résistance de l'élément augmente avec la diminution du rapport $\mathrm{D} / \mathrm{t}$. La capacité de déformation est non affectée.

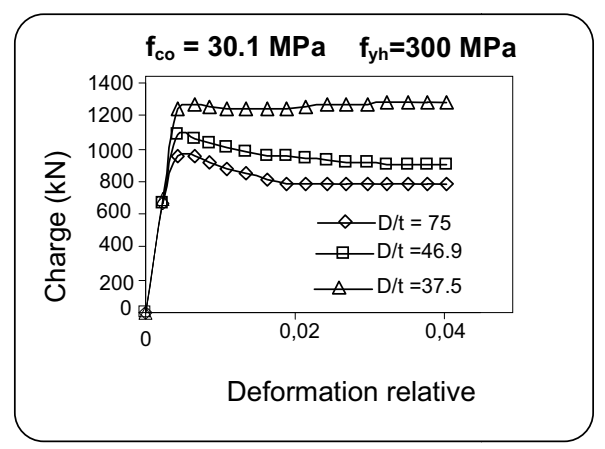

Fig. 2. Effet du rapport $\mathrm{D} / \mathrm{t}$

\section{Résumé de quelques modèles établis}

\subsection{Ehab et al. ${ }^{1}$}

L'approche utilisée dans l'établissement du modèle est similaire à celle de Mander et $a l^{3}{ }^{3}$ La contrainte pic de confinement et la déformation correspondante sont données par les expressions suivantes :

$$
f_{c c}=f_{c o}+4.1 f_{l} \quad \text { et } \quad \varepsilon_{c c}=\varepsilon_{c o}\left(1+20.5 \frac{f_{l}}{f_{c}}\right)
$$

(1)

Où $f_{l}$ représente la pression latérale de confinement imposée par le tube circulaire en acier.

Les expressions formulant la courbe contrainte déformation du béton confiné par le tube circulaire sont données par :

- pour la partie ascendante qui est d'ailleurs basée sur l'équation de Saenz ${ }^{4}$ :
$f_{c}=\frac{E_{c} \varepsilon_{c}}{1+\left(R+R_{E}-2\right) x-(2 R-1) x^{2}+R x^{3}}, \varepsilon_{c} \leq \varepsilon_{c c}$

avec $x=\frac{\varepsilon_{c}}{\varepsilon_{c c}}$ et $\mathrm{R}_{\mathrm{E}}$ et $\mathrm{R}$ sont respectivement données par les équations :

$R_{E}=\frac{E_{c} \varepsilon_{c c}}{f_{c c}}$ et $R=\frac{R_{E}\left(R_{\sigma}-1\right)}{\left(R_{\mathcal{\varepsilon}}-1\right)^{2}}-\frac{1}{R_{\varepsilon}}$

Selon $\mathrm{Hu}$ et al..$^{5}$, les constantes $\mathrm{R}_{\sigma}$ et $\mathrm{R}_{\varepsilon}$ sont prises égales à 4 .

- pour la partie descendante, le modèle opte pour une variation linéaire dictée par l'expression suivante :

$f_{c}=f_{c c}-\frac{\left(1-r k_{3}\right) f_{c c}}{10 \varepsilon_{c c}}\left(\varepsilon_{c}-\varepsilon_{c c}\right), \varepsilon_{c} \geq \varepsilon_{c c}$

Selon $\mathrm{Hu}$ et al. ${ }^{5}$, le coefficient $\mathrm{k}_{3}$ dépend du rapport $\mathrm{D} / \mathrm{t}$ et la limite élastique de l'acier $\mathrm{f}_{\mathrm{yh}}$. Le coefficient $\mathrm{r}$ est pris égal à 1 pour une résistance à la compression du béton inférieure à $30 \mathrm{MPa}$ et 0.5 pour des contraintes supérieures à $100 \mathrm{MPa}$. Pour les autres valeurs de contrainte on procède à une interpolation ${ }^{6}$.

\subsection{Han et al. ${ }^{7}$.}

Le confinement du béton est régi par les équations suivantes :

- Partie ascendante

$\frac{f_{c}}{f_{c c}}=2 x-x^{2}, \varepsilon_{c} \leq \varepsilon_{c c}$

- Partie descendante

$\frac{f_{c}}{f_{c c}}=\frac{x}{\beta(x-1)^{\eta}+x}, \varepsilon_{c} \geq \varepsilon_{c c}$

avec $x=\frac{\varepsilon_{c}}{\mathcal{E}_{c c}}$

La résistance pic est formulée comme suit :

$$
\begin{aligned}
& f_{c c}=\left[1+\left(-0.0135 \xi^{2}+0.1 \xi\right)\left(\frac{24}{f_{c}}\right)^{0.45}\right] f_{c} \\
& \text { où } \xi=\frac{A_{s} f_{y h}}{A_{c} f_{c o}}
\end{aligned}
$$

avec $\mathrm{A}_{\mathrm{s}}$ : section du tube d'acier, $\mathrm{f}_{\mathrm{co}}$ : résistance du béton La déformation correspondante à la résistance pic est exprimée par :

$$
\begin{aligned}
& \varepsilon_{c c}=\varepsilon_{c l}+\left[1330+760\left(\frac{f_{c}}{24}-1\right)\right] \xi^{0.2} \\
& \text { avec: } \varepsilon_{c l}=1300+12.5 f_{c}, \eta=1.6+1.5 / x
\end{aligned}
$$

et

$$
\beta= \begin{cases}\frac{f_{c}^{0.1}}{1.35 \sqrt{1 .+\varepsilon_{c o}}} & (\xi \leq 3.0) \\ \frac{f_{c}^{0.1}}{1.35 \sqrt{1 .+\varepsilon_{c o}}(\xi-2)^{2}} & (\xi \succ 3.0)\end{cases}
$$




\subsection{Susantha et al. ${ }^{2}$}

Le modèle proposé par l'auteur (2001), figure 3, est basé sur l'équation de Popovics ${ }^{8}$, qui ultérieurement a été modifiée par Mander et al. ${ }^{3}$.

Les équations régissant le modèle sont comme suit :

- branche ascendante : $\varepsilon_{\mathrm{c}} \leq \varepsilon_{\mathrm{cc}}$

$f_{c}=f_{c c}\left[\frac{r x}{r-1+x^{r}}\right]$

avec $\quad x=\frac{\varepsilon_{c}}{\varepsilon_{c c}}$ et

$$
r=\frac{E_{c}}{E_{c}-\frac{f_{c c}}{\varepsilon_{c c}}}
$$

et $f_{c c}=f_{c}+4 f_{r p}, \varepsilon_{c c}=\varepsilon_{c o}\left[1+5\left(\frac{f_{c c}}{f_{c}}-1\right)\right]$

La contrainte latérale de confinement est prise égale à :

$f_{r p}=\frac{2}{\frac{D}{t}-2} f_{y h}$

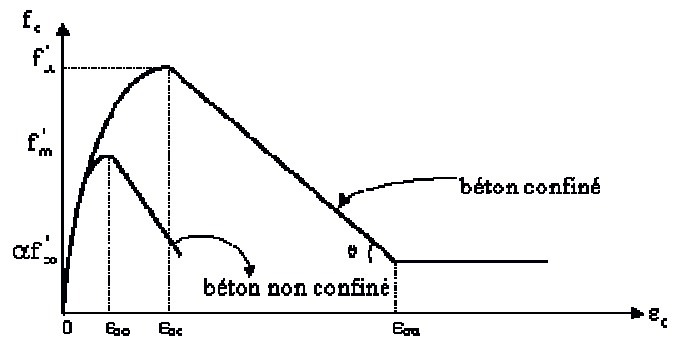

Fig. 3. Loi de comportement du béton selon Susantha

- branche descendante : $\mathcal{E}_{c} \geq \mathcal{E}_{c c}$

$f_{c}=f_{c c}-Z\left(\varepsilon_{c}-\varepsilon_{c c}\right)$

où $\mathrm{Z}$ est le taux d'adoucissement, qui est fonction des conditions suivantes :

$-\mathrm{Z}=0 \quad$ pour $R_{t}\left(f_{c o} / f_{y h}\right) \leq 0.006$

- $Z=1.0 \times 10^{5} R_{t} \frac{f_{c o}}{f_{y h}}-600$

pour $R_{t}\left(f_{c o} / f_{y h}\right) \geq 0.006$ et $f_{y h} \leq 283 \mathrm{MPa}$

$$
\text { - } \quad Z=1.0 \times 10^{5} R_{t} \frac{f_{c o}}{f_{y h}}-6000
$$

pour $R_{t}\left(f_{c o} / f_{y h}\right) \geq 0.006$ et $f_{y h} \geq 336 \mathrm{MPa}$

$-Z=\left(\frac{f_{y h}}{283}\right)^{13.4}\left[1.0 \times 10^{5} R_{t} \frac{f_{c o}}{f_{y h}}-600\right]$

pour

$R_{t}\left(f_{c o} / f_{y h}\right) \geq 0.006$ et $283 \mathrm{MPa} \leq f_{y h} \leq 336 \mathrm{MPa}$

avec $R_{t}=\sqrt{3\left(1-v^{2}\right)} \frac{f_{y h}}{E_{s}} \frac{D}{2 t}$

\section{Etude paramétrique selon le modèle de Susantha et al. ${ }^{2}$}

Les figures 4, 5 et 6 montrent analytiquement l'effet des principaux paramètres influents selon le modèle de Susantha. On remarque que le modèle reflète raisonnablement les résultats expérimentaux, en l'occurrence l'influence de $\mathrm{f}_{\mathrm{co}}$ et $\mathrm{D} / \mathrm{t}$. Concernant l'effet de la limite élastique sur la courbe contraintedéformation, on constate qu'il est bénéfique sur le comportement de l'élément.
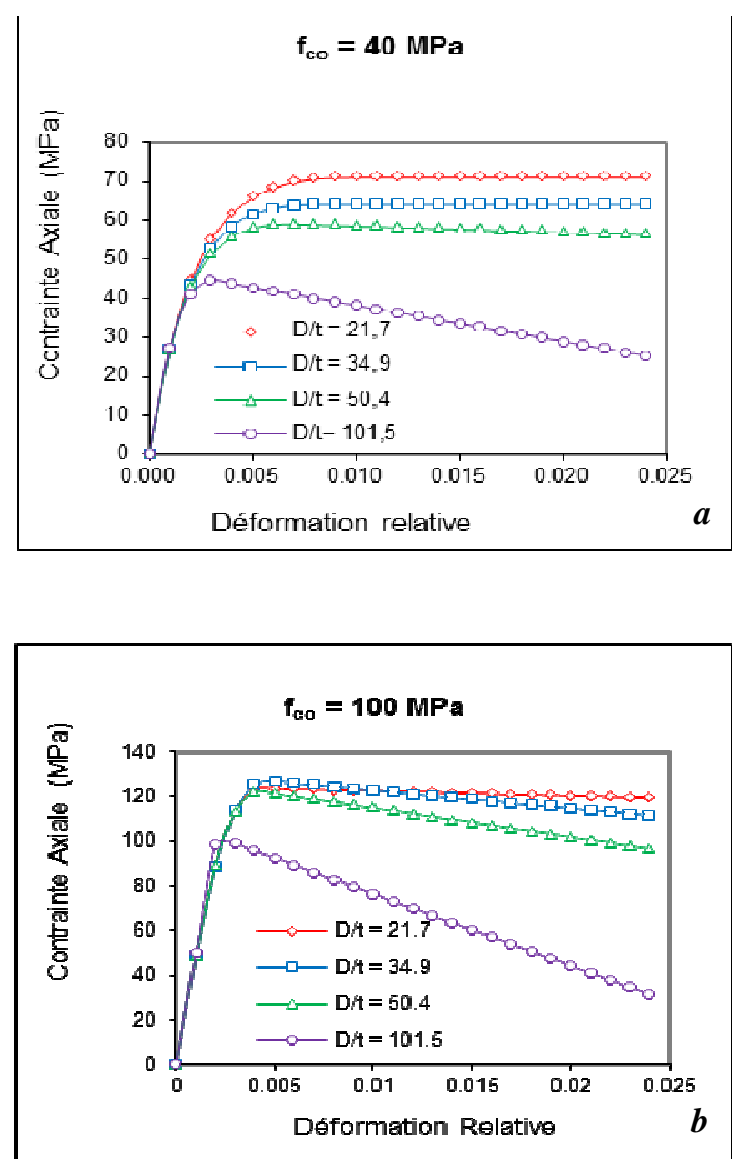

Fig. 4. Effet du rapport D/t

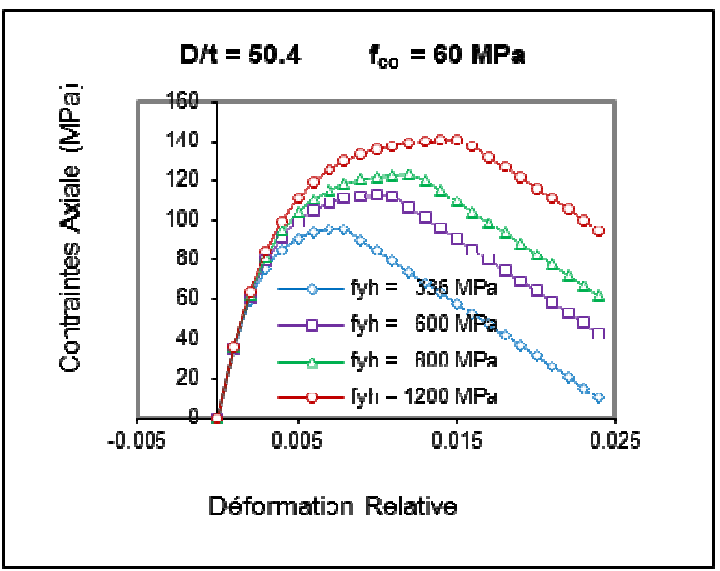

Fig. 5. Effet de la limite élastique de l'acier $\mathrm{f}_{\mathrm{yh}}$ 


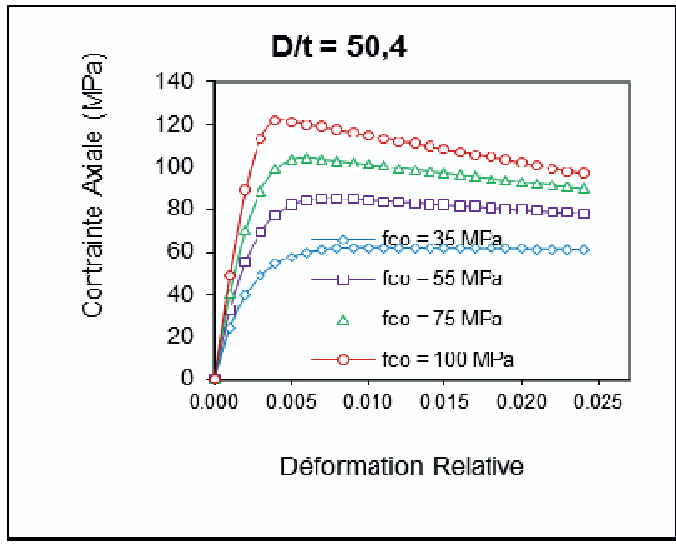

Fig. 6. Effet de la résistance du béton $\mathrm{f}_{\mathrm{co}}$

\subsection{Evaluation des paramètres de confinement}

De la relation contrainte-déformation, on définit les caractéristiques suivantes :

- Le gain en résistance, le paramètre $K_{s} \quad\left(K s=f_{c c} / f_{c o}\right)$ exprimant le rapport des contraintes pics des bétons confinés et non confiné ;

- Le gain en déformation, le paramètre $\mathrm{K}_{\mathrm{d}}\left(\mathrm{Kd}=\varepsilon_{\mathrm{cc}} / \varepsilon_{\mathrm{co}}\right)$ exprimant le rapport des déformations correspondantes respectivement aux contraintes pics du béton confiné et non confiné ;

- Gain en résistance

$K_{s}=1+4\left(f_{m} / f_{c}\right)=1+\frac{8}{\left(\frac{D}{t}-2\right)} \frac{f_{y h}}{f_{c}}$

Le coefficient $K_{s}$ est directement proportionnel à $f_{y h}$ et inversement proportionnel à $\mathrm{f}_{\mathrm{co}}$ et $\mathrm{D} / \mathrm{t}$.

- Gain en déformation

$K_{d}=\frac{\varepsilon_{c c}}{\varepsilon_{c o}}=\left[1+5\left(\frac{f_{c c}}{f_{c}}-1\right)\right]$

Le coefficient $K_{d}$ est directement proportionnel à $f_{c c}$ et inversement proportionnel à $\mathrm{f}_{\mathrm{co}}$.

\section{Détermination de la capacité axiale ultime $\mathbf{P}$}

Les figures 4, 5 et 6 montrent analytiquement l'effet des principaux paramètres influents selon le modèle de Susantha. On remarque que le modèle reflète raisonnablement les résultats expérimentaux, en l'occurrence l'influence de $\mathrm{f}_{\text {co }}$ et $\mathrm{D} / \mathrm{t}$. Concernant l'effet de la limite élastique sur la courbe contraintedéformation, on constate qu'il est bénéfique sur le comportement de l'élément.

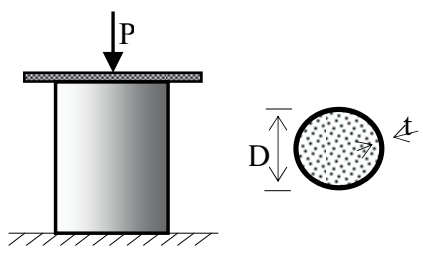

Fig. 7. Elément en tube d'acier soumis à une force de compression $\mathrm{P}$
L'équation de base permettant de déterminer la charge ultime est de la forme:

$P=P_{c}+P_{s}=A_{c} f_{c}+A_{s} f_{s}$

Dans cette équation les termes $\mathrm{A}_{\mathrm{c}}$ et $\mathrm{A}_{\mathrm{s}}$ sont supposés connus, ils représentent respectivement l'aire du béton et celle de l'acier enveloppant. Les contraintes $f_{c}$ et $f_{s}$ respectivement du béton et de l'acier, sont à déterminer. Pour résoudre cette équation, un code en Visual Basic a été développé s'appuyant sur l'algorithme suivant :

- Initialiser une valeur de $\mathrm{P}_{\text {ext }}$ (effort sollicitant l'élément).

- Initialiser des valeurs de D/t, D, $\mathrm{f}_{\mathrm{yh}}, \mathrm{f}_{\mathrm{co}}$.

- Initialiser une valeur de la déformation dans le béton $\varepsilon_{\mathrm{c}}$.

- Déterminer les contraintes dans le béton et l'acier $\left(f_{c}\right.$ et $f_{s}$ ) en utilisant les lois de comportement contraintes-déformations appropriées.

- Calculer l'effort axial P par l'équation 16.

- Comparer $\mathrm{P}_{\mathrm{ext}}$ et $\mathrm{P}$.

- Si l'équilibre entre les deux efforts, sollicitant et résistant est satisfaisant, la combinaison la combinaison $\mathrm{P}, \mathrm{D} / \mathrm{t}, \mathrm{D}, \mathrm{f}_{\mathrm{yh}}, \mathrm{f}_{\mathrm{co}}$ constitue une solution du problème et passer à l'étape 8 , sinon revenir à l'étape 3 et incrémenter la valeur de $\varepsilon_{c}$.

- Calculer la valeur de contrainte latérale de confinement $\mathrm{f}_{\mathrm{rp}}$.

- Incrémenter $\mathrm{P}_{\mathrm{ext}}$.

\subsection{Etude comparative et vérification des résultats}

Les tableaux 1 et 2 présentent les dimensions et propriétés des différents spécimens [Ehab et Young 2006], ainsi que les résultats relatifs aux différentes équations, y compris celle qui a été proposée dans cette étude. Les résultats traités par MEF dans le tableau 2 sont présentés par Ehab ${ }^{1}$ : l'élément finis est un solide tridimensionnel à 8 nœuds (C3D8), le programme d'analyse est ABAQUS. Les hypothèses utilisées : comportement à l'interface béton-tube modélisé ; courbes multi linéaires $\sigma-\varepsilon$ de l'acier utilisées; effets du béton et béton confiné pris en considération.

Tableau.1. Dimensions et propriétés des spécimens

\begin{tabular}{|c|c|c|c|c|c|c|}
\hline \multirow{2}{*}{ Spécimen } & \multicolumn{4}{|c|}{ Dimensions } & \multicolumn{2}{c|}{$\begin{array}{c}\text { Propriétés } \\
\text { des } \\
\text { matériaux }\end{array}$} \\
\cline { 2 - 7 } & $\begin{array}{c}\mathrm{L} \\
{[\mathrm{mm}]}\end{array}$ & $\begin{array}{c}\mathrm{D} \\
{[\mathrm{mm}]}\end{array}$ & $\begin{array}{c}\mathrm{t} \\
{[\mathrm{mm}]}\end{array}$ & $\mathrm{D} / \mathrm{t}$ & $\begin{array}{c}\mathrm{f}_{\mathrm{co}} \\
{[\mathrm{MPa}]}\end{array}$ & $\begin{array}{c}\mathrm{f}_{\mathrm{yh}} \\
{[\mathrm{MP}} \\
\mathrm{a}]\end{array}$ \\
\hline S1 & 300 & 114 & 7.6 & 15 & 30 & 343 \\
\hline S6 & 300 & 114 & 2.85 & 40 & 30 & 343 \\
\hline S11 & 300 & 114 & 2.07 & 55 & 30 & 343 \\
\hline S16 & 300 & 114 & 1.63 & 70 & 30 & 343 \\
\hline S21 & 714 & 238 & 11.90 & 20 & 30 & 507 \\
\hline S26 & 714 & 238 & 3.9 & 60 & 30 & 507 \\
\hline S31 & 1080 & 360 & 8.00 & 45 & 30 & 525 \\
\hline S36 & 1080 & 360 & 6.55 & 55 & 30 & 525 \\
\hline
\end{tabular}


Tableau.2. Dimensions et propriétés des spécimens

\begin{tabular}{|c|r|r|r|r|r|}
\hline \multirow{2}{*}{ Spécimen } & \multicolumn{5}{|c|}{ Capacité axiale ultime } \\
\cline { 2 - 6 } & \multirow{2}{*}{ Analyse } & \multicolumn{1}{c|}{ MEF } & \multicolumn{1}{c|}{$\mathbf{P}_{\text {EC4 }}$} & \multicolumn{1}{c|}{$\mathbf{P}_{\text {ACI }}$} & $\frac{\text { Analyse }}{\mathbf{P}_{\text {EC4 }}}$ \\
\hline S1 & 1339.0 & 1560.0 & 1554.2 & 1028.2 & 0.86 \\
\hline S6 & 812.5 & 757.5 & 798.5 & 529.5 & 1.02 \\
\hline S11 & 683.4 & 567.9 & 654.3 & 443.2 & 1.04 \\
\hline S16 & 594.2 & 491.3 & 459.1 & 329.7 & 1.29 \\
\hline S21 & 6488.3 & 7360.0 & 7782.3 & 5206.5 & 0.83 \\
\hline S26 & 3389.0 & 2932.0 & 3694.8 & 2540.9 & 0.92 \\
\hline S31 & 9466.0 & 8440.0 & 10391.5 & 7017.3 & 0.91 \\
\hline S36 & 8349.5 & 7200.0 & 9127.1 & 6231.0 & 0.91 \\
\hline
\end{tabular}

Les résultats obtenus sont confrontés aux différentes méthodes de calcul préconisées par l'Eurocode $4^{9}$ et $\mathrm{l}^{\prime} \mathrm{ACI}^{10}$, selon les équations suivantes:

$P_{E C 4}=A c f_{C}\left(1+\eta_{1} \frac{t}{D} \frac{f y}{f c}\right)+A_{S} f_{y h} \eta_{2}$

$A_{c}$ : aire de la section transversale du béton

$\mathrm{A}_{\mathrm{s}}$ : aire de la section transversale de l'acier de construction.

$\mathrm{f}_{\mathrm{c}}$ et $\mathrm{f}_{\mathrm{yh}}$ : résistances caractéristiques conformément à l'EC2 ou a l'EC3.

$\mathrm{t}$ : épaisseur de la paroi du tube en acier.

$\mathrm{d}$ : diamètre extérieur du poteau.

Les valeurs des coefficients $\eta_{1}$ et $\eta_{2}$ pour $0 \leq e \leq \frac{d}{10}$ :

$\eta_{1}=\eta_{10}\left(1-10 \frac{e}{d}\right) ; \eta_{2}=\eta_{20}+\left(1-\eta_{20}\right) 10 \frac{e}{d}$

Pour $e>\frac{e}{10} \quad \eta_{1}=0$ et $\eta_{2}=1.0$

e : excentrement du chargement

$\eta_{10}=4.9-18.5 \bar{\lambda}+17 \bar{\lambda}^{2} \quad($ mais $\geq 0)$

$\eta_{20}=0.25(3+2 \bar{\lambda})$

$($ mais $\leq 0)$

$\bar{\lambda}$ élancement non dimensionnel

$\mathrm{P}_{\mathrm{ACI}}=0.85 \mathrm{Acf}_{\mathrm{C}}+\mathrm{A}_{\mathrm{S}} \mathrm{f}_{\mathrm{yh}}$

La validation des résultats obtenus par l'analyse proposée selon le modèle de Susantha en comparaison avec l'Eurocode 4 montre une bonne concordance.

\subsection{Analyse des résultats et discussion}

Les figures 8, 9 et 10 montrent analytiquement l'effet des principaux paramètres sur la variation de la capacité ultime des tubes d'acier remplis de béton.

Les Figures 9a et $9 \mathrm{~b}$ montrent respectivement la variation de $f_{c o}$ avec la charge portante $P$ et la contrainte latérale de confinement $\mathrm{f}_{\mathrm{rp}}$ pour différentes valeurs de $\mathrm{D} / \mathrm{t}$.

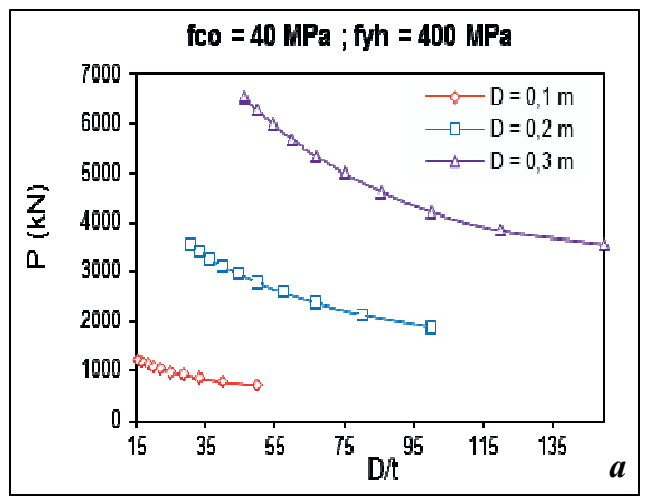

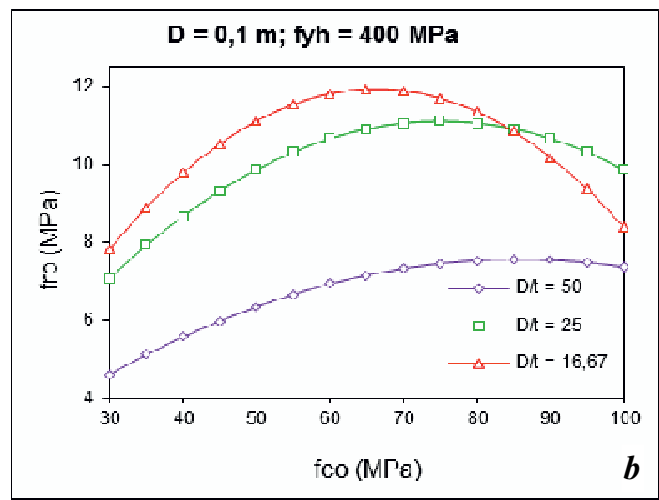

Fig. 8. Effet du rapport D/ t (suite)

\subsubsection{Effet de la résistance du béton $f_{\text {co }}$}

Sur la Figure 9a, la charge portante P croît avec la diminution de $\mathrm{D} / \mathrm{t}$ pour une valeur de $\mathrm{D}$ constante. Cependant le taux d'accroissement de la charge diminue avec la diminution de D/t. Sur la Figure 9b, la variation de la contrainte latérale de confinement croit avec l'augmentation de $\mathrm{f}_{\mathrm{co}}$ et le rapport $\mathrm{D} / \mathrm{t}$ jusqu'à une certaine valeur de $\mathrm{f}_{\text {co }}\left(\mathrm{f}_{\mathrm{co}} \approx 70 \mathrm{MPa}\right)$. Au delà de cette valeur, la contrainte latérale de confinement diminue avec l'accroissement de $\mathrm{f}_{\text {co }}$. Cette diminution devient de plus en plus significative avec le décroissement de $\mathrm{D} / \mathrm{t}$. Ces courbes mettent en évidence l'influence de l'interaction béton - tube d'acier : plus le béton est résistant, moindre est sa capacité de déformation transversale générant l'étreinte latérale.
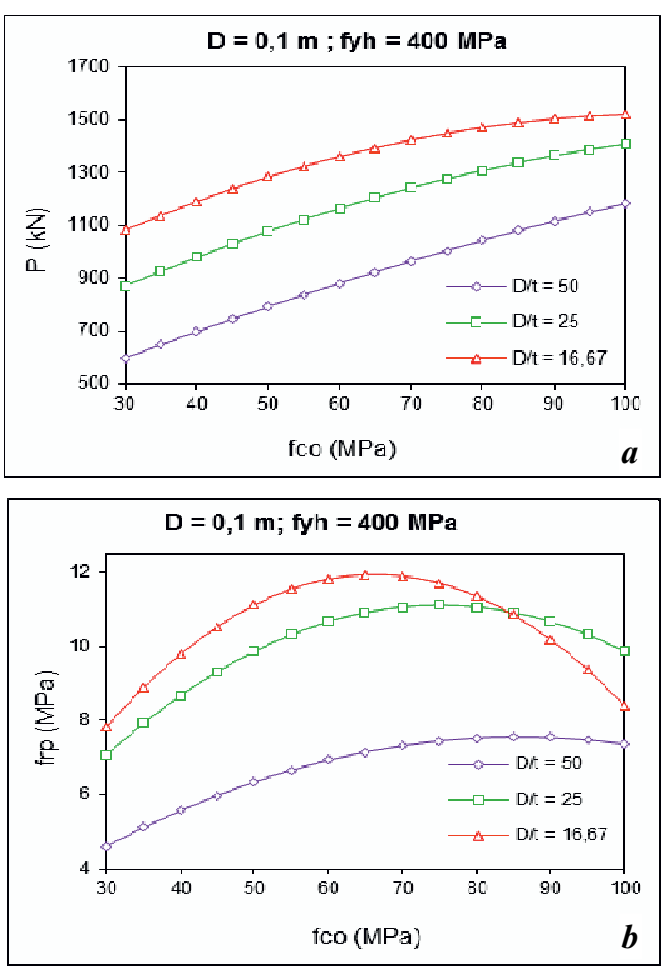

Fig. 9. Effet de la résistance du béton $\mathrm{f}_{\text {co }}$ 


\subsubsection{Effet de la limite élastique de l'acier $f_{y h}$}

Les Figures 10a et 10b montrent l'influence de la limite élastique de l'acier sur la charge portante $P$. Comme l'illustrent les Figures 10a et 10c, la charge augmente avec la limite élastique de l'acier utilisé ; cette augmentation est moins importante pour les rapports $\mathrm{D} / \mathrm{t}$ élevés. Elle devient insignifiante pour $\mathrm{D} / \mathrm{t}=100$ (Fig. 10c). L'effet de l'étreinte latérale est beaucoup plus prononcé dans le cas de D/t faible. L'examen des Figures $10 \mathrm{~b}$ et $10 \mathrm{~d}$ révèle que l'effet de la limite élastique de l'acier $f_{y h}$ sur la variation de l'étreinte latérale de confinement est plus important pour les petits diamètres et devient négligeable pour des rapports $\mathrm{D} / \mathrm{t}$ importants.
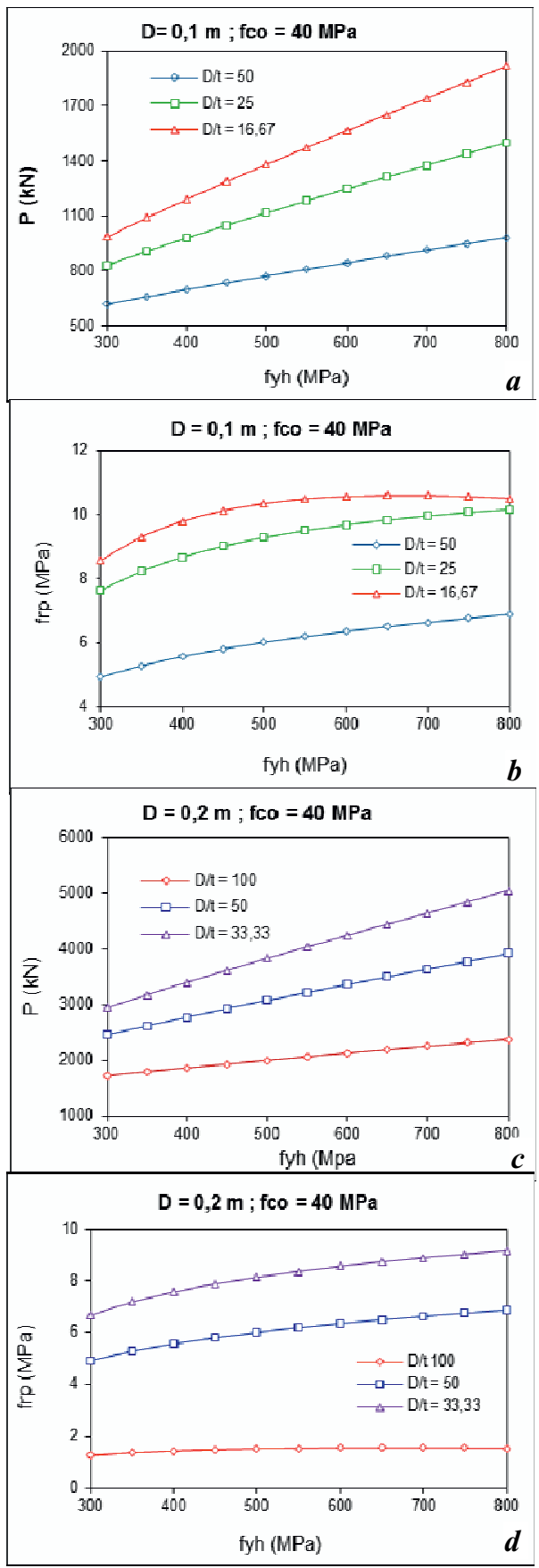

Fig. 10. Effet de la limite élastique de l'acier $\mathrm{f}_{\mathrm{yh}}$

\section{Conclusions}

Cette étude a permis d'émettre les points suivants :

- les paramètres majeurs influençant le comportement $\mathrm{du}$ béton confiné par tube d'acier qui sont: la résistance du béton, la limite élastique du tube d'acier et le rapport géométrique $\mathrm{D} / \mathrm{t}$.

- le gain en résistance augmente avec l'augmentation de la résistance du béton, diminue avec l'accroissement du rapport $\mathrm{D} / \mathrm{t}$ et croit proportionnellement avec la limite élastique de l'acier.

- le gain en déformation décroît avec l'augmentation de la résistance et est inaffecté par la variation du rapport $\mathrm{D} / \mathrm{t}$, ni par la variation de la résistance $\mathrm{du}$ béton.

Une équation directe pour l'analyse des tubes d'acier circulaires remplis de béton simple a été présentée. Le modèle de confinement a été parfaitement introduit. La prédiction des résultats obtenus a montré une bonne concordance avec ceux préconisés par les différents codes ainsi que ceux donnés par la méthode des éléments finis. Une étude paramétrique mettant en évidence l'influence des principaux paramètres, notamment le rapport $\mathrm{D} / \mathrm{t}$, la résistance du béton $f_{c o}$ et la limite élastique de l'acier transversal $f_{\text {yh }}$, a été menée. Les résultats analytiques ont montré l'effet favorable du confinement sur la capacité axiale ultime des éléments en TARB.

\section{References}

1. Ehab E., Young B., Behaviour of Normal Strength Concrete-Filled Compact Steel Tube Circular Stub Columns, Journal of Constructional Steel Research. 62 (2006) 706-715.

2. Susantha K. A. S., Hanbin G., Usami T., Uniaxial Stress-Strain Relationship of Concrete Confined by Various Shaped Steel Tubes, Engineering Structures, 23 (2001) 1331-1347.

3. Mander J. B., Priestley M. J. N., Park R., Theoretical Stress-Strain Model for Confined Concrete, Journal of Structural Engineering, Vol.114, 8, (1988), pp. 1804-1826.

4. Saenz LP, Discussion of Equation for Stress-Strain Curve of Concrete, by Desayi P. and Krishnan S., Journal of the American Institute, (1964), 1229 - 35.

5. Hu HT, Huang CS, Wu MH, Nonlinear Analysis of Axially Loaded Concrete - Filled Tube Columns with Confinement Effect, Journal of Structural Engineering, ASCE (2003); 129; 1322-9.

6. Tomii M., Ductile and Strong Columns Composed of Steel Tubes Infilled Concrete and Longitudinal Steel Bars, Proc., $3^{\text {rd }}$ Int. Conf. on Steel - Concrete Composite Structures, Fukuoka, Japan, (1991).

7. Han LH, Concrete - Filled Tube Columns, Science Publishing Company, (2004).

8. Popovics S., A Numerical Approach to the Complete Stress-Strain Curve Concrete, Cement and Concrete Research, vol. 3, (1973), pp. 583-599.

9. Eurocode 4. Design of composite steel and concrete structures. Part 1.1, General rules for buildings (with 
UK national application document) DD ENV 19941-1. London (UK): British Standards Institution; (1994).

10. Building code requirements for structural concrete and commentary, ACI 318-99. Detroit (USA): American Concrete Institute; (1999).

11. Giakoumelis G, Lam D. Axial capacity of circular concrete-filled tube columns. Journal of Constructional Research (2004), 60(7) : 1049-68.

12. Tomii M, Yoshimura K, Morishita Y. Experimental studies on concrete filled steel tubular stub columns under concentric load ing. In: International colloquium on stability of structures under static and dynamic load; Washington, DC, (1977) ; 718-41. 